\title{
Medizinische Praxiskoordinatorin: Ausbau der Interprofessionalität
}

\author{
Jürg Schlup \\ Dr. med., Präsident der FMH
}

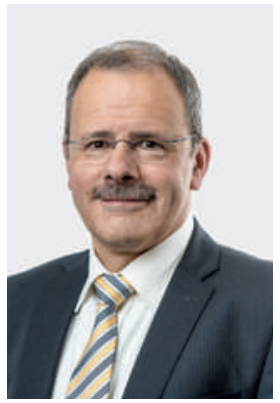

Wenn es um unsere Gesundheitsversorgung und deren zukünftige Herausforderungen geht, sind zwei Stichworte allgegenwärtig: Ärztemangel und Multimorbidität - letzteres insbesondere mit Blick auf chronische Erkrankungen und die demographische Entwicklung. Die Situation hat zu vielen Diskussionen unter Fachpersonen und Politikern Anlass gegeben, wie die Weiterentwicklung der interprofessionellen Zusammenarbeit zu einer verbesserten Gesundheitsversorgung beitragen könnte. Verschiedene Berufsgruppen versuchten ihre (vermeintlichen) Potentiale für die Sicherstellung der Grundversorgung aufzuzeigen und gesetzlich festschreiben zu lassen. So verwiesen die Apotheker bei der Revision des MedBG auf ihre Kompetenzen zur «Diagnose und Behandlung häufiger Krankheiten", und die Pflege beschreibt im Rahmen der parlamentarischen Initiative Joder ihr Selbstverständnis als «Drehscheibe der interdisziplinären Betreuung der Patienten».

\section{Da bei Erkrankungen die Arztpraxis}

die häufigste Anlaufstelle ist, spielt die MPA eine zentrale Rolle für die Zukunftsfähigkeit der Gesundheitsversorgung.

Erstaunlicherweise findet jedoch die Berufsgruppe der Medizinischen Praxisassistentinnen MPA in diesen Diskussionen meist keinerlei Beachtung. Symptomatisch hierfür ist, dass auch bei der Entwicklung der SAMWCharta zur «Zusammenarbeit der Fachleute im Gesundheitswesen» die MPA aussen vor blieben.

Dies ist umso unverständlicher, wenn man sich entscheidende Fakten der Gesundheitsversorgung vor Augen führt, wie z.B. dass der Grossteil aller medizinischen Behandlungen ambulant erfolgt. Ab dem Alter von 75 Jahren sind mehr als die Hälfte der Bevölkerung von mindestens einer chronischen Erkrankung und etwa ein Drittel von Multimorbidität betroffen. Patienten dieser Altersgruppe gehen durchschnittlich vier Mal im Jahr zum Hausarzt - benötigen bis zum 90. Lebensjahr jedoch mehrheitlich keine Pflegeleistungen.

Mit dem Inkrafttreten des Prüfungsreglements für Medizinische Praxiskoordinatorinnen (MPK) im Fe-

bruar 2015 wird somit eine Weiterbildungsmöglichkeit für MPAs geschaffen, die umsetzt, was auf der Hand liegt: die MPA als erste Ansprechperson in der Arztpraxis in die Betreuung dieser wachsenden und teilweise sehr viel Zeit erfordernden Patientengruppe einzubeziehen. So können MPKs mit klinischer Fachrichtung die ambulante Ärzteschaft in grossem Umfang und sinnvoll entlasten, Qualitätseinbussen der Behandlung sind durch die hohe Qualifikation sowie die enge $\mathrm{Zu}$ sammenarbeit in der Praxis hingegen nicht zu befürchten.

Ein weiterer unbestrittener und wichtiger Trend in der Gesundheitsversorgung ist die Zunahme von grösseren ambulanten Behandlungszentren, die mit neuen organisatorischen Erfordernissen einhergehen. Diesem wird die MPK mit praxisleitender Fachrichtung gerecht, die in Praxismanagement und Personalführung spezifisch geschult ist - und auch angesichts der überbordenden Administration im Gesundheitswesen zu einer Entlastung der Ärzteschaft führen dürfte.

Das neue Berufsbild der MPK holt die MPA aus der beruflichen Sackgasse und verdeutlicht nicht zuletzt auch die Offenheit der Ärzteschaft für Veränderungen bestehender Rollen- und Aufgabenverteilungen. Der lange Weg zur nun in Kraft getretenen Prüfungsordnung für MPKs zeigt, wie eine gute Weiterentwicklung der interprofessionellen Zusammenarbeit unter Einbezug verschiedener Gesundheitsberufe aussehen sollte. Mehrere MPA- und Ärzteverbände haben sich gemeinsam bei der Erarbeitung dieser Weiterbildungsmöglichkeit eingebracht. Die Schnittstellen zu anderen Berufsgruppen, wie Pflegenden oder Ernährungsbera-

MPK mit klinischer und praxisleitender Fachrichtung können Ärztinnen und Ärzte spürbar entlasten.

terinnen, wurden intensiv bearbeitet und konsensuell bereinigt. Das Ergebnis ist ein für alle Beteiligte attraktives neues Tätigkeitsfeld, das einen spürbaren Beitrag zur Zukunftsfähigkeit unserer Gesundheitsversorgung leisten kann. Die FMH wünscht den ersten und allen weiteren Kandidatinnen der Berufsprüfung viel Erfolg! 